\title{
Rectangle Covers Revisited Computationally
}

\author{
LAURA HEINRICH-LITAN ${ }^{1}$ and MARCO E. LÜBBECKE ${ }^{2}$
}

\begin{abstract}
We consider the problem of covering an orthogonal polygon with a minimum number of axisparallel rectangles from a computational point of view. We propose an integer program which is the first general approach to obtain provably optimal solutions to this well-studied $\mathcal{N} \mathcal{P}$-hard problem. It applies to common variants like covering only the corners or the boundary of the polygon, and also to the weighted case. In experiments it turns out that the linear programming relaxation is extremely tight, and rounding a fractional solution is an immediate high quality heuristic. We obtain excellent experimental results for polygons originating from VLSI design, fax data sheets, black and white images, and for random instances. Making use of the dual linear program, we propose a stronger lower bound on the optimum, namely the cardinality of a fractional stable set. Furthermore, we outline ideas how to make use of this bound in primal-dual based algorithms. We give partial results which make us believe that our proposals have a strong potential to settle the main open problem in the area: To find a constant factor approximation algorithm for the rectangle cover problem.
\end{abstract}

Categories and Subject Descriptors: F.2.2 [Analysis of Algorithms and Problem Complexity]: Nonnumerical Algorithms and Problems-Geometrical problems and computations

General Terms: Algorithms, Experimentation

Additional Key Words and Phrases: Linear Programming, Integer Programming

\section{INTRODUCTION}

A polygon with all edges either horizontal or vertical is called orthogonal. Given an orthogonal polygon $P$, the rectangle cover problem is to find a minimum number of possibly overlapping axis-parallel rectangles whose union is exactly $P$. In computational geometry, this problem received considerable attention in the past 25 years, in particular with respect to its complexity and approximability in a number of variants. Still, the intriguing main open question [Bern and Eppstein 1997] is:

Is there a constant factor approximation algorithm for the rectangle cover problem?

An extended abstract of this paper appeared in the Proceedings of the 4th International Workshop on Efficient and Experimental Algorithms (WEA05) [Heinrich-Litan and Lübbecke 2005].

${ }^{1}$ Advanced Driver Information Technology GmbH, Robert-Bosch-Str. 200, D-31132 Hildesheim, Germany, lheinrich-litan@de.adit-jv.com

${ }^{2}$ Technische Universität Berlin, Institut für Mathematik, Sekr. MA 6-1, Straße des 17. Juni 136, D-10623 Berlin, Germany, m.luebbecke@math.tu-berlin.de.

Permission to make digital/hard copy of all or part of this material without fee for personal or classroom use provided that the copies are not made or distributed for profit or commercial advantage, the ACM copyright/server notice, the title of the publication, and its date appear, and notice is given that copying is by permission of the ACM, Inc. To copy otherwise, to republish, to post on servers, or to redistribute to lists requires prior specific permission and/or a fee.

(c) $20 \mathrm{YY}$ ACM 0000-0000/20YY/0000-0001 $\$ 5.00$ 
We do not answer this question now, but we offer a different and new kind of reply, which is "computationally, yes." In fact, we provide a fresh experimental view, the first of its kind, on the problem which has applications in the fabrication of masks in the design of DNA chip arrays [Hannenhalli et al. 2002], in VLSI design, and in data compression, in particular in image compression.

Previous work. Customarily, one thinks of the polygon $P$ as a union of finitely many (combinatorial) pixels, sometimes also called a polyomino. The polygon $P$ can be associated with a visibility graph $G$ [Maire 1994; Motwani et al. 1989a; 1989b; Schrijver 2003]: The vertex set of $G$ is the set of pixels of $P$ and two vertices are adjacent in $G$ if and only if their associated pixels can be covered by a common rectangle. Rectangles correspond to cliques in $G$. A clique is a set of vertices, any two of which are adjacent. We will denote by $\theta$ the number of rectangles in an optimal cover. An obvious lower bound on $\theta$ is the size $\alpha$ of a maximum stable set in $G$, also called maximum independent set. This is a set of pixels, no two of which are contained in a common rectangle. In the literature one also finds the notion of an antirectangle set.

Chvátal originally conjectured that $\alpha=\theta$, and this is true for convex polygons [Chaiken et al. 1981] and a number of special cases. Szemerédi gave an example with $\theta \neq \alpha$, see Fig. 1. Intimately related to the initially stated open question, Erdős then asked whether $\theta / \alpha$ was bounded by a constant. In [Chaiken et al. 1981] an example is mentioned with $\theta / \alpha \geq 21 / 17-\varepsilon$, however, this example cannot be reconstructed from [Chaiken et al. 1981], and thus we cannot verify it. The best proven bound is $\theta / \alpha \geq 8 / 7$.

For polygons with holes and even for those without holes (also called simple polygons) the rectangle cover problem is $\mathcal{N} \mathcal{P}$-hard [Masek 1979; Culberson and Reckhow 1994] and Max $\mathcal{S N} \mathcal{N}$-hard [Berman and DasGupta 1997]. In particular this implies that there is no polynomial time approximation scheme. Since the rectangle cover problem is a special set cover problem, the standard greedy approach immediately gives an $O(\log n)$ approximation. For general polygons, Anil Kumar and Ramesh [2003] are the first (and currently best) to improve upon this obvious factor, giving an approximation guarantee of $O(\sqrt{\log n})$, where $n$ is the number of edges of $P$. The proof of this result is rather involved. For simple polygons Franzblau [1989] gives a sweepline algorithm which achieves an approximation factor of 2. In the general case, the proven factor of this algorithm is $O(\log n)$ but conjectured by Franzblau [1989] to be 3. The lower bounds used to prove approximation guarantees are the stable set size $\alpha$ and (an upper bound on) the size of a largest clique in $G$. A constant factor approximation in the general case can be considered as the remaining white spot in the problem's complexity landscape.

Quite some research efforts have gone into finding polynomially solvable special cases; we mention only covering with squares [Aupperle et al. 1988; Levcopoulos and Gudmundsson 1997] and polygons in general position [Bern and Eppstein 1997]. Interestingly, there is a polynomial time algorithm for partitioning a polygon into non-overlapping rectangles [Ohtsuki 1982]. However, a polygon similar to Fig. 4 shows that an optimal partition size may exceed an optimal cover size by a linear factor, so this does not lead to a constant factor approximation. 
Our Contributions. Despite its theoretical hardness, we demonstrate the rectangle cover problem to be computationally very tractable. In particular we study an integer programming formulation of the problem. Doing this, we are the first to offer an exact (of course non-polynomial time) algorithm to obtain provably optimal solutions, and we are the first to introduce linear/integer programming techniques in this problem area. It has been pointed out e.g., by Anil Kumar and Ramesh [2003] that the main difficulty in coming up with good approximation algorithms is to find good lower bounds on the optimum cover size. Based on a fractional solution to the (dual of the) linear programming relaxation we propose a stronger lower bound which we call the fractional stable set size. In fact, this new lower bound motivates us to pursue previously unexplored research directions to find a constant factor approximation algorithm. These are the celebrated primal-dual scheme [Goemans and Williamson 1996], rounding a fractional solution, iterated rounding [Jain 2001], and a dual fitting algorithm [Vazirani 2001].

Currently with "only" encouraging computational results in our hands, we are optimistic that our research will actually contribute to a positive answer to the initially stated long standing open question. We sketch partial results and promising ideas. A fruitful contribution of our work is a number of open questions it spawns. Last but not least we hope to increase the awareness in the computational geometry community towards tools from mathematical programming.

Preliminaries. Since we are dealing with a combinatorial problem, we identify $P$ with its set of combinatorial pixels. This way we write $p \in P$ to state that pixel $p$ is contained in polygon $P$. Let $R$ denote the set of all rectangles in $P$. It is important that we only count rectangles and do not consider their areas. Thus, it is no loss of generality to restrict attention to inclusionwise maximal rectangles. We will do so in the following without further reference. The number of these rectangles can still be quadratic in the number $n$ of edges of $P$ [Franzblau 1989], see also Fig. 2.

\section{AN INTEGER PROGRAM}

Interpreting rectangles as cliques in $G$ we can make use of the standard integer programming formulation for the minimum clique cover problem in graphs [Schrijver 2003]. A binary variable $x_{r}$ indicates whether rectangle $r \in R$ is chosen in the cover or not. For every pixel $p \in P$ at least one rectangle which covers $p$ has to be picked, and the number of picked rectangles has to be minimized:

$$
\begin{array}{ll}
\theta=\min \sum_{r \in R} x_{r} & \\
\text { s. t. } \sum_{r \in R: r \ni p} x_{r} \geq 1 \quad & p \in P \\
x_{r} \in\{0,1\} \quad r \in R
\end{array}
$$

This integer program (which we call the primal program) allows us to optimally solve any given instance of our problem, and we will do so in our experiments. When we replace (3) by $x_{r} \geq 0, r \in R\left(3^{\prime}\right)$, we obtain the associated linear programming (LP) relaxation. There is no need to explicitly require $x_{r} \leq 1, r \in R$, since we 
are minimizing. We call the optimal objective function value of the LP relaxation the fractional cover size of $P$ and denote it by $\bar{\theta}$. Clearly, it holds that $\bar{\theta} \leq \theta$. In general, no polynomial time algorithm is known to compute the fractional clique cover number of a graph, that is, for solving this linear program [Schrijver 2003]. In our case, however, the number of variables and constraints is polynomial in $n$, in fact quadratic, due to the fact that we work with maximal rectangles only. Therefore, the fractional cover size $\bar{\theta}$ can be computed in polynomial time.

The integer program (1)-(3) immediately generalizes to the weighted rectangle cover problem, where rectangles need not have unit cost. It is straightforward, and it does not increase the complexity, to restrict the coverage requirement to particular features of the polygon like the corners or the boundary - two well-studied variants [Berman and DasGupta 1997] for which no exact algorithm has been presented. It is also no coincidence that a formal dualization of our program leads to a formulation for the dual problem of finding a maximum stable set. A binary variable $y_{p}, p \in P$, reflects whether a pixel is chosen in the stable set or not. We have to require that no rectangle contains more than one of the chosen pixels, and we maximize the number of chosen pixels. We call this the dual integer program:

$$
\begin{aligned}
& \alpha=\max \sum_{p \in P} y_{p} \\
& \text { s. t. } \sum_{p \in P: p \in r} y_{p} \leq 1 \quad r \in R \\
& y_{p} \in\{0,1\} \quad p \in P
\end{aligned}
$$

Again, when replacing (6) by $y_{p} \geq 0, p \in P\left(6^{\prime}\right)$, we obtain the associated LP relaxation. We call its optimal objective function value $\bar{\alpha}$ the fractional stable set size of $P$. We refer to a feasible solution to the dual as a fractional stable set. It holds that $\bar{\alpha} \geq \alpha$. By strong linear programming duality we have $\bar{\alpha}=$ $\bar{\theta}$. We stress again the fact that we distinguish between the (primal and dual) integer programs which solve the problems exactly, and their respective continuous linear programming relaxations, which give bounds. In general, optimal solutions to both linear programs (1)-(3') and $(4)-\left(6^{\prime}\right)$ are fractional. However, using an interesting link to graph theory, in the case that $G$ is perfect [Golumbic 1980], optimal solutions are automatically integer because of a strong duality between the integer programs [Schrijver 2003]. This link was established already early, see e.g., [Berge et al. 1981; Motwani et al. 1989a; 1989b], and our linear programs give optimal integer covers in polynomial time for this important class of polygons with $\alpha=\theta$.

\subsection{About Fractional Solutions}

Our computational experiments fuel our intuition. Anticipating our numerical results we already give some qualitative observations here. In linear programming based approximation algorithms the objective function value of a primal or dual fractional solution is used as a lower bound on the integer optimum. The more we learn about such fractional solutions the more tools we may have to analyze the problem's approximability. 

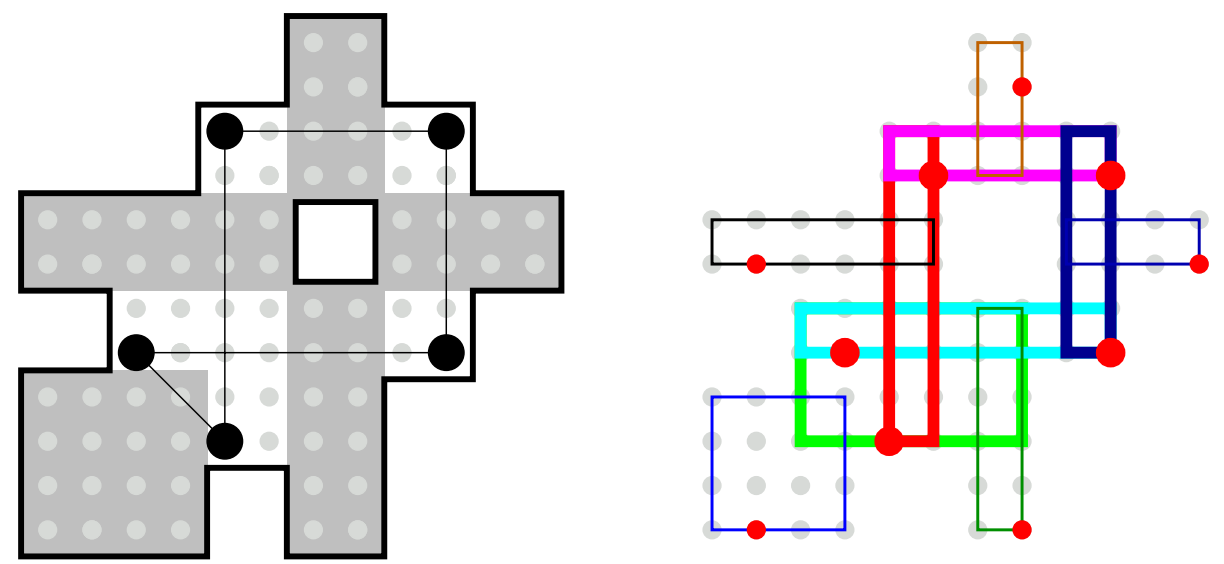

Fig. 1. The original counterexample to $\alpha=\theta$ by Szemerédi and (to the right; indicated is the odd whole) an optimal fractional cover. Thicker lines (points) indicate rectangles (pixels) which are picked to the extent of 0.5 .

General Observations. The linear relaxations $(1)-\left(3^{\prime}\right)$ and $(4)-\left(6^{\prime}\right)$ appear to be easily solvable to optimality in a few seconds on a standard PC. The vast majority of variables already assumes an integer value. A mere rounding of the remaining fractional variables typically gives an optimal or near-optimal integer solution (e.g., instance night is a bad example with "only" $95 \%$ integer values, but the rounded solution is optimal). For smaller random polygons the LP optimal solution is very often already integer; and this is an excellent quality practical heuristic, though memory expensive for very large instances.

Odd Holes. Fig. 1 (left) shows Szemerédi's counterexample to the $\alpha=\theta$ conjecture. The 5 rectangles indicated by the shaded parts have to be in any cover. In the remaining parts of the polygon, there are 5 pixels which induce an odd-length cycle $C$ ("odd hole") in the visibility graph $G$. To cover these pixels, at least 3 rectangles are needed, implying $\theta \geq 8$. On the other hand, at most 2 of these pixels can be independent, that is, $\alpha \leq 7$. The odd hole $C$ is precisely the reason why $G$ is not perfect in this example. Fig. 1 (right) shows that $C$ is encoded in the optimal fractional solution as well: Exactly the variables corresponding to edges of $C$ assume a value of 0.5 . The same figure shows an optimal fractional stable set. Pixels corresponding to vertices of $C$ assume a value of 0.5 (drawn fatter in the figure). That is, $\bar{\alpha}=\bar{\theta}=7.5$. This immediately suggests to strengthen the LP relaxation.

Lemma 2.1. For any induced odd cycle $C$ with $|C| \geq 5$, the inequality $\sum_{r \in C} x_{r} \geq$ $\lceil|C| / 2\rceil$ is valid for (1)-(3), where $r \in C$ denotes the rectangles corresponding to the edges of $C$.

The graph theoretic complements of odd holes are called odd antiholes. A graph is not perfect either if it contains an induced odd antihole. We can prove that there is no way of representing even the simplest non-trivial antihole with 7 vertices in a 
rectangle visibility graph. Odd holes are therefore the only reason for imperfection, however, they are not the only reason for fractional solutions. From our experiments, arbitrary fractions are possible, not only halves. More explicitly, we know of no way of lower bounding the smallest occurring fraction, and simply rounding a fractional solution does not give a constant factor approximation. We pursue this a little further next.

High Coverage. We define the coverage of a pixel $p$ as the number of rectangles which contain $p$. For the classical set cover problem, rounding up an optimal fractional solution gives an $f$-approximate cover, where $f$ is the maximum coverage of any element. In general, a pixel can have more than constant coverage; even worse, almost no pixel may have constant coverage. Even in an optimal cover of a simple polygon in general position pixels may have high coverage (see Fig. 2). Unlike in the general set cover case, high coverage is no prediction about the fractions in an optimal LP solution: In Fig. 2 (right) there are no fractional variables, the solution is integer. The fractional (indeed integer) optimal solution to this simple example has a remarkable property. Every rectangle in the optimal cover contains pixels of low coverage. More precisely, the following holds.

Lemma 2.2. In an optimal cover $\mathcal{C}$, every rectangle $r \in \mathcal{C}$ contains a pixel which is uniquely covered by $r$.

This can be easily seen since otherwise $\mathcal{C} \backslash\{r\}$ would be a cover, contradicting the optimality of $\mathcal{C}$. We call these uniquely covered pixels private. Since the number of private pixels is the number of rectangles in a cover we deem this aspect of the problem worthy of more investigation. Even more so since by complementary slackness (see below) private pixels (these fulfill the constraints (5) with equality) are the only candidates for being picked (at least fractionally) into a stable set.

Note that a set of private pixels need not be a stable set. It is therefore natural to relax the concept and ask: What are the characteristics of polygons where every pixel has only constant coverage? What kind of polygons have "many" pixels with "low" coverage? How can we exploit Lemma 2.2? Answers to these questions would turn LP rounding into a constant factor approximation algorithm. These questions also keep appearing in different guises in the next section.

\section{LP BASED APPROXIMATION}

There are more elaborate linear programming based approaches to constant factor approximation algorithms. They can be used as analytical tools to theoretically sustain our excellent computational results.

\subsection{Iterated Rounding}

In standard LP rounding (of all the fractional variables) the approximation factor hinges on the magnitude of the smallest fraction. Alternatively, Jain [2001] proposed to round up only one fractional variable at a time, adapt the linear program to reflect the rounding, solve the modified linear program again, and iterate. Adapting the linear program in our case means that rounding up a rectangle $r$ at 

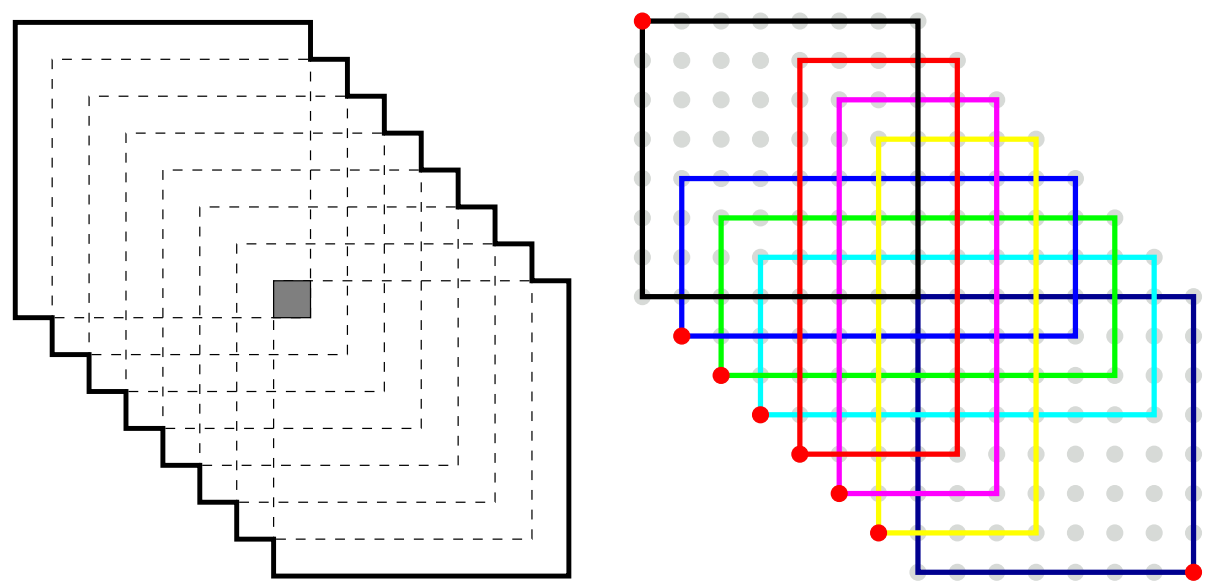

Fig. 2. Left: The shaded center pixel is covered by any maximal rectangle; almost all pixels have non-constant coverage. In an optimal cover, the coverage of the center pixel is linear in the cover size. The right figure schematically shows a minimal cover and a maximum stable set. Note that there are pixels of small, in fact unique coverage in the cover-we call these private.

fractional value $x$ is the same as reducing the coverage requirement for all the pixels in $r$ from 1 to $1-x$.

Proving an approximation factor via this iterated rounding scheme relies on two parts: The first is that one can always (in every iteration) find a rectangle/variable with a large value, and the second is that a solution to the final (modified) linear program is still feasible to the initial linear program in order to guarantee that the initial linear program actually gives a lower bound on the cover size of the rounded solution. The latter is indeed immediate for our linear program. For the former, in our experiments we always found (fractional) variables of value at least 0.5 , and a geometric argument why this is always the case would be most interesting.

\subsection{Primal-Dual Scheme}

The primal-dual scheme [Goemans and Williamson 1996] builds on relaxing the complementary slackness optimality conditions [Schrijver 2003] in linear programming. The general scheme iteratively improves an initially infeasible integer primal solution, that is, a set of rectangles, to finally obtain a feasible cover. The improvement step is guided by a feasible fractional dual solution, that is a fractional stable set, which is improved in alternation with the primal solution. The relaxed complementary slackness conditions contain the key information. In our case they read

$$
x_{r}>0 \Rightarrow \frac{1}{d} \leq \sum_{p \in P: p \in r} y_{p} \quad r \in R
$$

for some constant $d$, and

$$
y_{p}>0 \Rightarrow \sum_{r \in R: r \ni p} x_{r} \leq c \quad p \in P
$$


for some constant $c$. First note that if a possibly infeasible primal integer solution is maintained, $x_{r}>0$ means $x_{r}=1$. An interpretation of condition (7) is that every rectangle in the constructed cover must cover at least $1 / d$ pixels from the fractional stable set. Condition (8) states that a pixel in the fractional stable set must not be contained in more than $c$ rectangles (regardless of whether in the cover or not).

We found two cases where we can compute a cover and a fractional stable set simultaneously such that the two conditions hold. Thin polygons, as unions of width 1 or height 1 rectangles, are a class of polygons amenable to LP rounding and the primal-dual scheme: Since no pixel is covered by more than two rectangles this gives a 2-approximation. More generally, polygons of bounded width (every pixel contains a boundary pixel in its "neighborhood") are a new non-trivial class which allows a constant factor approximation. Of course, the primal-dual scheme trivially applies when all rectangles have bounded (combinatorial) area.

\subsection{Dual Fitting}

Since $\alpha \leq \theta$ the former natural approach to approximation algorithms was to construct a large stable set usable as a good lower bound [Franzblau 1989]. Since $\alpha \leq \bar{\alpha}$ we propose to use the stronger bound provided by a fractional stable set. Our dual fitting approach is to simultaneously construct a cover $\mathcal{C} \subseteq R$ and an pseudo stable set $\mathcal{S} \subseteq P$ of pixels with $|\mathcal{C}| \leq|\mathcal{S}|$ (we say that $\mathcal{S}$ pays for $\mathcal{C}$ ). "Pseudo" refers to allowing a constant number $c$ of pixels in a rectangle, that is, we relax (5) to $\sum_{p \in P: p \in r} y_{p} \leq c$. From this constraint we see that picking each pixel in $\mathcal{S}$ to the extent of $1 / c$ (which is a division of all $y_{p}$ variables' values by $c$ ) gives a feasible fractional solution to our dual linear program. A cover with these properties has a cost of

$$
|\mathcal{C}| \leq|\mathcal{S}| \leq c \cdot \bar{\alpha}=c \cdot \bar{\theta} \leq c \cdot \theta,
$$

that is, it would yield a $c$-approximation. Actually, one does not have to require that $\mathcal{S}$ pays for the full cover but $\frac{1}{d}|\mathcal{C}| \leq|\mathcal{S}|$ for a constant $d$ suffices, which would imply a $(c \cdot d)$-approximation. This paying for a constant fraction of the primal solution only is a new proposal in the context of dual fitting. Here again, the question is how to guarantee our conditions in general. From a computational point of view, we obtain encouraging results which suggest that our proposal can be developed into a proven constant factor approximation. In the next section we sketch some ideas how this can be done.

\section{TOWARDS A CONSTANT FACTOR APPROXIMATION}

\subsection{Obligatory Rectangles and Greedy}

For set cover, the greedy algorithm yields the best possible approximation factor of $O(\log n)$. The strategy is to iteratively pick a rectangle which covers the most yet uncovered pixels. One expects that for our particular problem, the performance guarantee can be improved. Computationally, we answer strictly in the affirmative. Again, our contribution is the dual point of view. It is our aim to design an algorithm which is based on the dual fitting idea of Section 3.3, and we mainly 
have to say how to construct a feasible dual fractional solution.

We use some terminology from [Hannenhalli et al. 2002]. Certain rectangles have to be in any cover. A prime rectangle contains a pixel which is not contained in any other rectangle. Such a pixel is called a leaf. Every cover must contain all prime rectangles. For a given pixel $p$ we may extend horizontally and vertically until we hit the boundary; the rectangular area $R(p)$ defined by the corresponding edges at the boundary is called the extended rectangle of $p . R(p)$ might not be entirely contained in the polygon but if so, it is a prime rectangle [Hannenhalli et al. 2002]. Moreover, let $\mathcal{C}^{\prime} \subseteq \mathcal{C}$ be a subset of some optimal cover $\mathcal{C}$. If there is a rectangle $r$ which contains $\left(P \backslash \mathcal{C}^{\prime}\right) \cap R(p)$ for some extended rectangle $R(p)$, then there is an optimal cover which contains $\mathcal{C}^{\prime}$ and $r$ [Hannenhalli et al. 2002]. In this context, let us call rectangle $r$ quasi-prime and pixel $p$ a quasi-leaf. The algorithm we use to compute a cover is a slight extension of [Hannenhalli et al. 2002], but we will provide a new interpretation, and more importantly, a dual counterpart:

\section{QuAsI-GREEDY}

1. pick all prime rectangles

2. pick a maximal set of quasi-prime rectangles

3. cover the remaining pixels with the greedy algorithm

4. remove redundant rectangles ("pruning")

It has not been observed before that a set of leafs and quasi-leafs forms a stable set. This leads to the idea to compute a pseudo stable set containing a maximal set of leafs and quasi-leafs. Thus, in order to build a pseudo stable set we check for every rectangle in the greedy cover whether it contains

1. a leaf

2. a quasi-leaf

3. a corner pixel

(in this order). The first positive test gives a pixel which we add to the pseudo stable set. A corner pixel is a corner of a rectangle which is private and a corner of the polygon. We already observed that pixels from steps 1 and 2 are independent. Furthermore, any rectangle obviously contains at most 4 corner pixels, and since corner pixels are private, actually at most 2 of them. By our previous considerations, this would imply a 2-approximation if the constructed pseudo stable set would pay for the whole cover. In general, we found this not to be true. We have constructed examples which suggest that one cannot guarantee that a constant fraction of the cover has been paid for. To achieve this latter goal one has to add more pixels to the pseudo stable set. To this end we extend the above test and also check for every rectangle in the cover whether it contains

\section{4. a border pixel.}

A border pixel $p$ is private and adjacent to a non-polygon pixel $\bar{p}$ (the outer face or a hole). The row (or column) of pixels which contains $p$, which is adjacent to $\bar{p}$, and which extends to the left and the right (to the top and the bottom) until some non-polygon pixel is hit must not be adjacent to a different hole (or the outer 


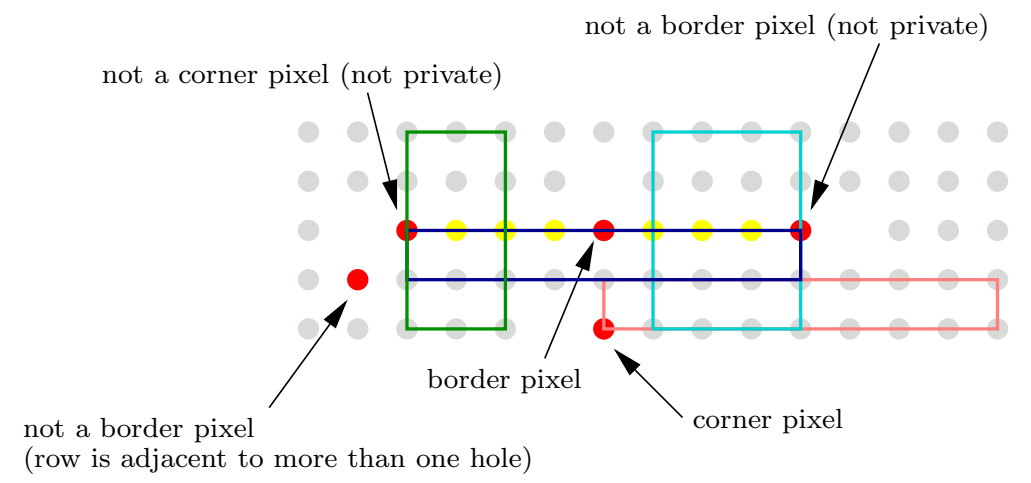

Fig. 3. Illustrating corner and border pixels; yellow pixels are the row of the border pixel (which is adjacent to only one hole).

face) other than the hole (or the outer face) the pixel $\bar{p}$ corresponds to, see Fig. 3. Also these pixels have a natural motivation: They are a kind of analogue to corner pixels in the sense that a border pixel represents an edge of a rectangle. It is, however, conceivable that some rectangle contains several border pixels picked into the pseudo stable set, even though we conjecture than one cannot come up with a geometry that enforces such a situation.

Let us furthermore remark that after the pruning step in QUASI-GREEDY, every rectangle in the cover contains a private pixel (Lemma 2.2). This pixel is an intuitive candidate to become a pixel in a pseudo stable set. This set would actually pay for the whole cover. However, it is not clear whether one can control how many pixels of this set can be contained in the same rectangle.

\subsection{Using Boundary Covers}

There is a simple 4-approximation algorithm for covering the boundary of an orthogonal polygon [Berman and DasGupta 1997]. In this context a natural question arises: Can we always find an interior cover whose size is bounded from above by a constant multiple of the size $\theta_{\text {boundary }}$ of an optimal boundary cover? The answer is "no." Our counterexample in Fig. 4 shows that there is an $O(\sqrt{n})$-cover of the boundary of the polygon in the left figure with maximal horizontal and vertical strips. But the optimal interior cover needs $\Theta(n)$ rectangles since the white uncovered pixels in the right figure are independent. Nevertheless, the latter observation is actually very encouraging. We conjecture that one can find an interior cover of size less than $c_{1} \cdot \theta_{\text {boundary }}+c_{2} \cdot \alpha$ where $c_{1}$ are $c_{2}$ are appropriate constants. This would imply a constant factor approximation for the rectangle cover problem.

We constructed an example (Fig. 5) that shows that an optimal boundary cover may leave an odd hole of interior pixels uncovered. This implies that a solution to a linear program for covering the remaining pixels may be fractional. 

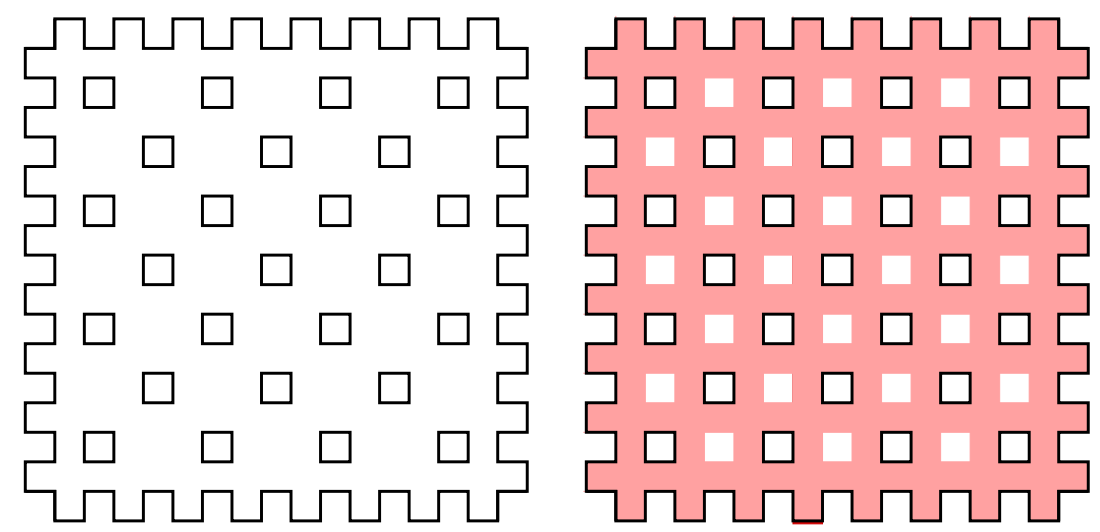

Fig. 4. A boundary cover may leave a non-constant fraction of pixels uncovered. Note that in this example all uncovered pixels are leafs, i.e., uniquely contained in some rectangle and therefore are all independent. A (4-approximation of a) boundary cover and a cover for these pixels obtained e.g., by our QUASI-GREEDY algorithm is thus a proven 5-approximation (in fact, actually optimal).
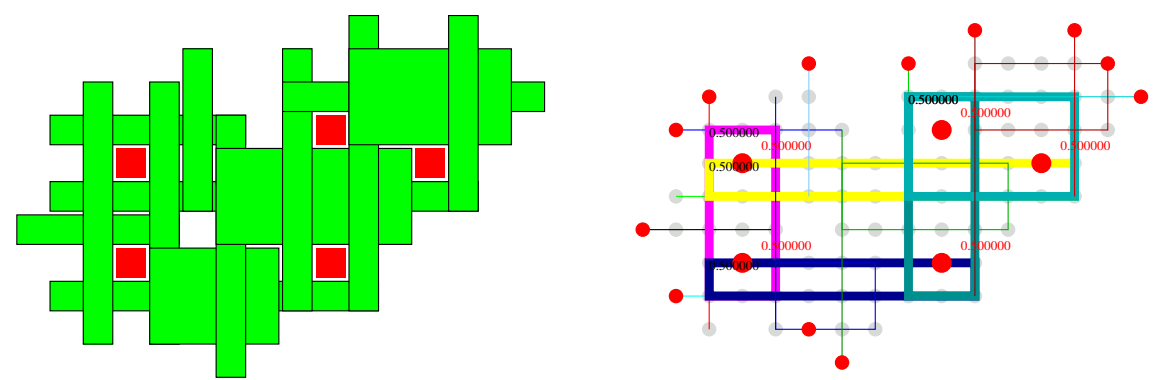

Fig. 5. An optimal boundary cover of this polygon is depicted with green rectangles; the red pixels form an odd hole which need to be covered by three additional rectangles. A fractional solution to the linear program is given on the right.

\subsection{Quasi-Prime Rectangles and Breaking Holes}

There is a large class of polygons (e.g., polygons resulting from typical oligonucleotide masks [Hannenhalli et al. 2002]) where the optimal cover is found after the first two steps of the QUASI-GREEDY algorithm in Section 4.1. Then the cover consists of only prime and quasi-prime rectangles. This is of course in general not the case (see Fig. 1). Now, consider the set $\mathcal{U}$ of pixels remained uncovered after step 2. We can prove that there is an induced cycle (a hole) in $G$ whose vertices correspond to a subset of $\mathcal{U}$. Covering each second edge of this hole extends the previous partial cover. We call this covering step to "break a hole." A straightforward algorithm is the following: while the polygon is uncovered, iteratively pick a maximal set of quasi-prime rectangles, then find a hole and break it. We can iteratively extend also the partial pseudo stable set. The quasi-prime rectangles are paid for by quasi-leafs, which form a stable set. The rectangles which break an even (odd) hole can all (but one) be paid for by a stable set, too. 
We have experimented with related and extended ideas based on the observations sketched in Sections 4.2 and 4.3 and obtained encouraging results. We hope that our findings will constitute an important step to enable researchers to finally come up with a constant factor approximation algorithm.

\section{COMPUTATIONAL EXPERIENCE}

A technical investigation of our LP based proposals for possible constant factor approximations for the rectangle cover problem and their computational evaluation went hand in hand. Directly or indirectly all primal-dual related questions boil down to "is it geometrically possible to select many pixels which are almost independent?" In this respect we think that the QUASI-GREEDY algorithm is a representative method to provide partial answers. It is also our aim to further sustain the somewhat unexpected well-behavior of the problem or our formulation.

\section{1 "Real-World" Polygons}

Our test set comprises polygons of various sizes which are derived from VLSI mask design (instances VLSI*), a set of standard fax images ${ }^{3}$ (instances ccitt*), and several black and white images (instances marbles, mickey,...). The ratio between the number of available rectangles and the optimal cover size can be seen as one indicator about the difficulty of an instance (this has to be used carefully as Fig. 2 shows an easy instance with $\theta^{2}$ rectangles). Tables I and II summarize our results.

\subsection{Random Polygons}

We used two different generators for random polygons. The first, flip-coin, simply decides for each pixel (of a square of given size) with a given probability (the same for every pixel) whether it is in the polygon or not. The second, union, generates a given number of rectangles and outputs their union: Each generated rectangle has random upper left and lower right corners (up to a given maximum side length, common for width and height), i.e., it is placed at a random position. Random always refers to uniformly at random. Fig. 6 shows sample polygons, Figs. 7 and 8 try to capture the difficulty of our random polygons. Tables III and IV summarize our results.

\subsection{Results}

Tables I and III give overwhelming evidence for the excellent quality of the lower bound obtained from the LP relaxation of our integer program. The integrality gap (relative gap between linear and integer program in percent) is always close to zero (for 1564 out of 1800 random polygons the gap actually is zero).

From Tables II and IV we first observe that covers computed with our QUASIGREEDY algorithm are generally very close to optimum. To computationally support our conjecture that the dual fitting analysis of QUASI-GREEDY indeed leads

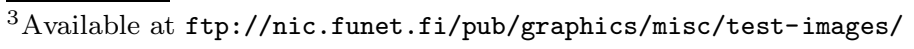



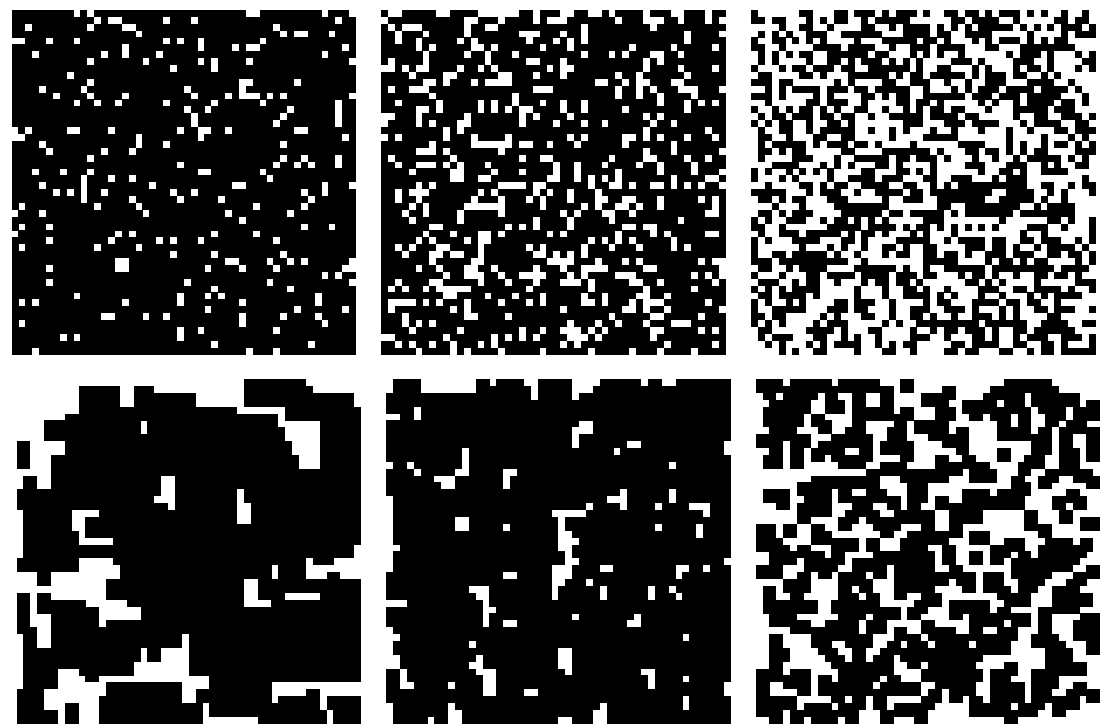

Fig. 6. Samples of random $50 \times 50$ polygons. The top row is generated with the flip-coin generator with pixel probabilities of $90 \%, 75 \%$, and $50 \%$. The bottom row is generated with the union generator: the characteristics as (maximal side length, number of rectangles) pairs are $(10,1000)$, $(5,2000)$, and $(3,2000)$, in that order. Even though union type polygons in the bottom row look "more natural" our algorithms are more challenged by dense flip-coin polygons.

to a constant approximation factor we evaluate several more characteristics of the pseudo stable sets we compute. Our findings are summarized in Figs. 9-14.

We have never seen more than 4 pixels of a pseudo stable set in a rectangle (which appears to be a sensible number to aim at in a geometry context). We remark that our observed worst case is 4 pixels but the average fraction of rectangles containing 3 or 4 pseudo stable pixels is almost negligibly small. Our pseudo stable sets consistently pay for more than $75 \%$ of the cover size. According to our line of argumentation in Section 4.1, this empirically supports that QUASI-GREEDY be an $\left(4 \cdot \frac{4}{3}\right)$-approximation algorithm for the rectangle cover problem. From Table IV it appears that large and dense polygons obtained from the flip-coin generator come closest to refute this conjecture; we generated density $90 \%$ instances of size up to $1000 \times 1000$, however, to no negative effect to our conjecture.

\subsection{Corner and Boundary Covers}

Finding a minimum cover only for the corners or the boundary of a polygon is $\mathcal{N} \mathcal{P}$ hard as well [Berman and DasGupta 1997]. Just out of interest, and to initiate a fresh thinking here as well, we computationally related the optimum cover sizes for these problems to minimum rectangle (interior) cover sizes.

The relation $\theta_{\text {corner }} \leq \theta_{\text {boundary }} \leq \theta$ is obvious. In computations we found that $\theta_{\text {boundary }}$ is closer to $\theta$ than to $\theta_{\text {corner }}$, and it appears that $\theta \leq 2 \cdot \theta_{\text {corner }}$ which implied that $\theta \leq 2 \cdot \theta_{\text {boundary }}$. Recall that there is a 4 -approximation for the boundary cover problem. 


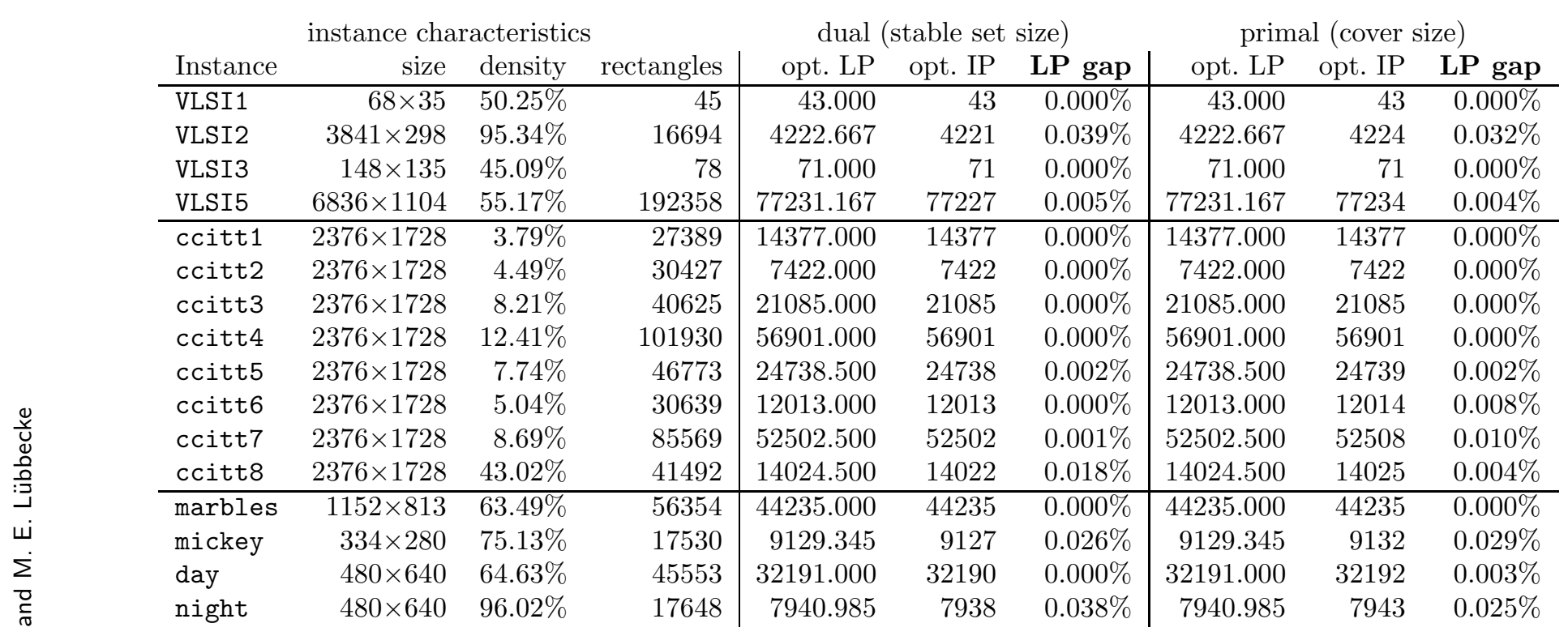

Table I. Results for the primal and dual linear/integer programs. For each instance we list its size in pixels, its number of pixels (as a fraction), and its number of (maximal) rectangles. For the dual and the primal programs (in that order) we give the optimal linear and integer program objective function values. The 'LP gap' is the relative gap between linear and integer program. Notice I. that instances mickey and night do not have a fractional optimal solution with 'nice' fractions. The 21086 reported as optimal for ccitt3 in the extended abstract [Heinrich-Litan and Lübbecke 2005] is improved here since the optimality tolerance of the solver was erroneously set to a non-zero value by default. 
QUASI-GREEY cover

pseudo stable set characteristics

\begin{tabular}{lrrr|rrr|rrrr} 
Instance & optimum & \multicolumn{2}{c}{ QUASI-GREEDY } & prime & quasi-prime & greedy & corner & border & max pixels & pays for \\
\hline VLSI1 & 43 & 43 & 1.000 & 41 & 2 & 0 & 0 & 0 & $100.00 \%$ \\
VLSI2 & 4224 & 4701 & 1.113 & 1587 & 203 & 2911 & 1105 & 1279 & 4 & $88.79 \%$ \\
VLSI3 & 71 & 71 & 1.000 & 71 & 0 & 0 & 0 & 0 & 1 & $100.00 \%$ \\
\hline ccitt1 & 14377 & 14457 & 1.006 & 10685 & 2099 & 1673 & 1632 & 28 & 2 & $99.91 \%$ \\
ccitt2 & 7422 & 7617 & 1.026 & 3587 & 409 & 3621 & 3574 & 29 & 3 & $99.76 \%$ \\
ccitt3 & 21086 & 21259 & 1.008 & 15691 & 2020 & 3548 & 3427 & 86 & 3 & $99.84 \%$ \\
ccitt4 & 56901 & 57262 & 1.006 & 42358 & 8605 & 6299 & 6110 & 59 & 2 & $99.77 \%$ \\
ccitt5 & 24739 & 24911 & 1.007 & 18529 & 2985 & 3397 & 3259 & 98 & 2 & $99.84 \%$ \\
ccitt6 & 12014 & 12132 & 1.010 & 8256 & 1049 & 2827 & 2764 & 35 & 2 & $99.77 \%$ \\
ccitt7 & 52508 & 52599 & 1.002 & 39230 & 10842 & 2525 & 2448 & 56 & 2 & $99.96 \%$ \\
ccitt8 & 14025 & 14303 & 1.020 & 7840 & 1353 & 5110 & 5023 & 54 & 3 & $99.77 \%$ \\
\hline marbles & 44235 & 44235 & 1.000 & 43548 & 687 & 0 & 0 & 0 & 1 & $100.00 \%$ \\
mickey & 9132 & 9523 & 1.043 & 5582 & 690 & 3251 & 528 & 1593 & 3 & $88.13 \%$ \\
day & 32192 & 32431 & 1.007 & 26308 & 3777 & 2346 & 749 & 900 & 4 & $97.85 \%$ \\
night & 7943 & 8384 & 1.056 & 4014 & 501 & 3869 & 762 & 1810 & 4 & $84.53 \%$
\end{tabular}

Table II. Details for the QUASI-GREEDY algorithm of Section 4.1. We compare the optimal cover size against ours: We list the absolute numbers and the relative quality of QUASI-GREEDY covers as a factor from optimum; this is typically around 1.01. The following columns list the number of prime and quasi-prime rectangles, and those picked by the greedy step. Then, the number of corner and border pixels in the constructed quasi stable set $\mathcal{S}$ is given (the number of (quasi-)leafs equals the number of (quasi-)primes). Finally, we state the maximal number of pixels of $\mathcal{S}$ in some rectangle, and the fraction of the cover size for which $\mathcal{S}$ pays. 


\begin{tabular}{l|rr|rr|rr} 
& & & primal & dual & & \\
Instance generator & density & rectangles & LP gap & LP gap & QUASI-GREEDY \\
\hline flip-coin $(50,90 \%)$ & 90.059 & 498.890 & .012 & .014 & 227.490 & 1.031 \\
flip-coin $(50,80 \%)$ & 79.966 & 626.190 & .000 & .000 & 364.550 & 1.005 \\
flip-coin $(50,70 \%)$ & 69.999 & 663.700 & .002 & .002 & 457.830 & 1.000 \\
flip-coin $(100,90 \%)$ & 90.013 & 2013.160 & .029 & .029 & 887.190 & 1.048 \\
flip-coin $(100,80 \%)$ & 80.008 & 2506.970 & .003 & .003 & 1430.310 & 1.008 \\
flip-coin $(100,70 \%)$ & 69.960 & 2661.090 & .001 & .001 & 1806.960 & 1.000 \\
flip-coin $(200,90 \%)$ & 90.031 & 8075.250 & .037 & .037 & 3506.940 & 1.058 \\
flip-coin $(200,80 \%)$ & 79.971 & 10045.080 & .004 & .004 & 5677.470 & 1.009 \\
flip-coin $(200,70 \%)$ & 69.974 & 10612.130 & .000 & .000 & 7166.130 & 1.001 \\
\hline union $(50,10,1000)$ & 91.630 & 65.360 & .000 & .000 & 30.780 & 1.005 \\
union $(50,5,2000)$ & 79.967 & 225.710 & .014 & .014 & 98.260 & 1.021 \\
union $(50,3,2000)$ & 72.162 & 364.020 & .009 & .009 & 172.130 & 1.022 \\
union $(100,20,1000)$ & 91.173 & 89.910 & .000 & .000 & 42.060 & 1.002 \\
union $(100,10,3000)$ & 86.760 & 352.440 & .018 & .018 & 137.100 & 1.027 \\
union $(100,6,4000)$ & 76.995 & 976.780 & .015 & .015 & 403.070 & 1.039 \\
union $(200,30,1500)$ & 89.397 & 244.130 & .006 & .006 & 94.250 & 1.016 \\
union $(200,15,5000)$ & 85.595 & 852.010 & .009 & .012 & 299.810 & 1.044 \\
union $(200,9,6000)$ & 61.749 & 2272.620 & .005 & .005 & 1038.140 & 1.016 \\
& & & & & &
\end{tabular}

Table III. Cumulated results for our experiments with random polygons. We give the characteristics for flip-coin polygons as (size, density) pairs and those for union polygons as triples (size, maximal side length, number of rectangles). We generated 100 polygons for each line in this table, 1800 in total. Headings' meanings are identical to those in Table I.

\section{CONCLUSIONS}

The rectangle cover problem turns out to be computationally very tractable. The extremely small integrality gaps are a strong vote for our integer programming approach. Despite such successes and the ability to obtain provably optimal solutions to geometric problems this methodology is not very common in computational geometry. We hope to contribute with our work to more curiosity towards mathematical programming techniques in this community. On the downside of it, integer programs for industrial size polygons, e.g., from VLSI design are extremely large. The generation of the integer programs consumes much more time than solving them (solution time is typically only a few seconds using the standard solver CPLEX 9.1.0 [ILOG Inc., CPLEX Division 2004]). As a remedy we also experimented with a column generation approach, that is, a dynamic generation of the variables of the linear program. This enables us to attack larger instances.

It is common that theory is complemented by computational experience. In this paper we did the reverse: We found promising research directions by a careful study of computational experiments. Finally, we propose:

Restatement of Erdös' Question. Is it true that both, the integrality gap of our primal and that of our dual integer program are bounded by a constant? The example in Fig. 1 places lower bounds on these gaps of $\theta / \bar{\theta} \geq 16 / 15$ and $\bar{\alpha} / \alpha \geq$ $15 / 14$, implying the already known bound $\theta / \alpha \geq 8 / 7$. We conjecture that these gaps are in fact tight. Originally, we set out to find an answer to Erdös' question. We conclude with an answer in the affirmative, at least computationally. 
QUASI-GREEY cover characteristics

pseudo stable set characteristics

\begin{tabular}{l|rrrr|rrrr} 
Instance generator & QUASI-GREEDY & prime & quasi-prime & greedy & corner & border & max pixels & pays for \\
\hline flip-coin $(50,90 \%)$ & 227.490 & 106.190 & 45.470 & 77.310 & 20.170 & 33.700 & 2.420 & 90.333 \\
flip-coin $(50,80 \%)$ & 364.550 & 240.820 & 90.740 & 33.490 & 15.380 & 10.910 & 2.060 & 98.157 \\
flip-coin $(50,70 \%)$ & 457.830 & 363.100 & 88.310 & 6.460 & 4.010 & 1.530 & 1.560 & 99.807 \\
flip-coin $(100,90 \%)$ & 887.190 & 372.720 & 105.690 & 417.820 & 109.110 & 173.280 & 2.980 & 85.747 \\
flip-coin $(100,80 \%)$ & 1430.310 & 912.340 & 331.680 & 188.580 & 84.190 & 61.130 & 2.320 & 97.133 \\
flip-coin $(100,70 \%)$ & 1806.960 & 1407.230 & 367.510 & 32.430 & 19.810 & 7.830 & 2.010 & 99.746 \\
flip-coin $(200,90 \%)$ & 3506.940 & 1381.700 & 292.770 & 1875.160 & 489.380 & 767.020 & 3.060 & 83.573 \\
flip-coin $(200,80 \%)$ & 5677.470 & 3556.520 & 1270.610 & 859.720 & 390.540 & 278.280 & 2.890 & 96.802 \\
flip-coin $(200,70 \%)$ & 7166.130 & 5551.210 & 1473.220 & 142.640 & 86.490 & 34.520 & 2.070 & 99.711 \\
\hline union $(50,10,1000)$ & 30.780 & 21.660 & 6.160 & 2.970 & 1.260 & 1.430 & 1.290 & 99.371 \\
union $(50,5,2000)$ & 98.260 & 59.720 & 17.050 & 21.900 & 8.670 & 10.280 & 1.840 & 97.166 \\
union $(50,3,2000)$ & 172.130 & 103.870 & 32.660 & 36.200 & 14.420 & 14.670 & 1.920 & 95.386 \\
union $(100,20,1000)$ & 42.060 & 30.550 & 6.720 & 4.870 & 2.520 & $2.000)$ & 1.380 & 99.534 \\
union $(100,10,3000)$ & 137.100 & 80.030 & 20.470 & 37.520 & 14.850 & 17.790 & 2.050 & 96.819 \\
union $(100,6,4000)$ & 403.070 & 219.120 & 62.500 & 124.500 & 47.750 & 53.690 & 2.380 & 93.931 \\
union $(200,30,1500)$ & 94.250 & 59.460 & 13.240 & 21.900 & 11.090 & 9.150 & 1.860 & 98.658 \\
union $(200,15,5000)$ & 299.810 & 166.190 & 33.830 & 102.380 & 40.820 & 48.550 & 2.510 & 96.086 \\
union $(200,9,6000)$ & 1038.140 & 691.800 & 154.850 & 195.540 & 92.980 & 75.340 & 2.080 & 97.825
\end{tabular}

Table IV. Cumulated results for our random experiments with polygons of flip-coin and union type. Headings' meanings are identical to those in Table II. Large and dense instances of this type appear to be most critical to refute our conjecture that our dual fitting approach leads to a constant factor approximation algorithm; see our comments in the text. 
Acknowledgments. We thank Sándor Fekete for fruitful discussions and Ulrich Brenner for providing us with polygon data from the mask fabrication process in VLSI design.

\section{REFERENCES}

Anil Kumar, V. And Ramesh, H. 2003. Covering rectilinear polygons with axis-parallel rectangles. SIAM J. Comput. 32, 6, 1509-1541.

Aupperle, L., Conn, H., Keil, J., And O’Rourke, J. 1988. Covering orthogonal polygons with squares. In Proc. 26th Allerton Conf. Commun. Control Comput. 97-106.

Berge, C., Chen, C., Chvátal, V., and Seow, C. 1981. Combinatorial properties of polyominoes. Combinatorica 1, 217-224.

Berman, P. And DasGupta, B. 1997. Complexities of efficient solutions of rectilinear polygon cover problems. Algorithmica 17, 4, 331-356.

Bern, M. AND Eppstein, D. 1997. Approximation algorithms for geometric problems. See Hochbaum [1996], Chapter 8, 296-345.

Chaiken, S., Kleitman, D., Saks, M., and Shearer, J. 1981. Covering regions by rectangles. SIAM J. Algebraic Discrete Methods 2, 394-410.

Culberson, J. and Reckhow, R. 1994. Covering polygons is hard. J. Algorithms 17, 2-44.

FranzBlau, D. 1989. Performance guarantees on a sweep-line heuristic for covering rectilinear polygons with rectangles. SIAM J. Discrete Math. 2, 3, 307-321.

Goemans, M. And Williamson, D. 1996. The primal-dual method for approximation algorithms and it application to network design problems. See Hochbaum [1996], Chapter 4.

Golumbic, M. 1980. Algorithmic Graph Theory and Perfect Graphs. Academic Press, New York.

Hannenhalli, S., Hubell, E., Lipshutz, R., And Pevzner, P. 2002. Combinatorial algorithms for design of DNA arrays. Adv. Biochem. Eng. Biotechnol. 7\%, 1-19.

Heinrich-LitAn, L. AND LüBBECKE, M. 2005. Rectangle covers revisited computationally. In Proceedings of the 4th International Workshop on Efficient and Experimental Algorithms (WEA05), S. Nikoletseas, Ed. Lect. Notes Comput. Sci., vol. 3503. Springer-Verlag, Berlin, 55-66.

Hochbaum, D., Ed. 1996. Approximation Algorithms for $\mathcal{N} \mathcal{P}$-Hard Problems. PWS Publishing Co., Boston, MA.

ILOG Inc., CPLEX Division. 2004. CPLEX 9.0 User's Manual.

JAIN, K. 2001. A factor 2 approximation algorithm for the generalized Steiner network problem. Combinatorica 21, 1, 39-60.

Levcopoulos, C. And Gudmundsson, J. 1997. Approximation algorithms for covering polygons with squares and similar problems. In Proceedings of RANDOM'9\%. Lect. Notes Comput. Sci., vol. 1269. Springer, Berlin, 27-41.

Maire, F. 1994. Polyominos and perfect graphs. Inform. Process. Lett. 50, 2, 57-61.

MASEK, W. 1979. Some NP-complete set covering problems. Unpublished manuscript, MIT.

Motwani, R., Raghunathan, A., and Saran, H. 1989a. Covering orthogonal polygons with star polygons: The perfect graph approach. J. Comput. System Sci. 40, 19-48.

Motwani, R., Raghunathan, A., And Saran, H. 1989b. Perfect graphs and orthogonally convex covers. SIAM J. Discrete Math. 2, 371-392.

Ohtsuki, T. 1982. Minimum dissection of rectilinear regions. In Proc. 1982 IEEE Symp. on Circuits and Systems, Rome. 1210-1213.

Schrijver, A. 2003. Combinatorial Optimization: Polyhedra and Efficiency. Springer, Berlin.

Vazirani, V. 2001. Approximation Algorithms. Springer, Berlin. 


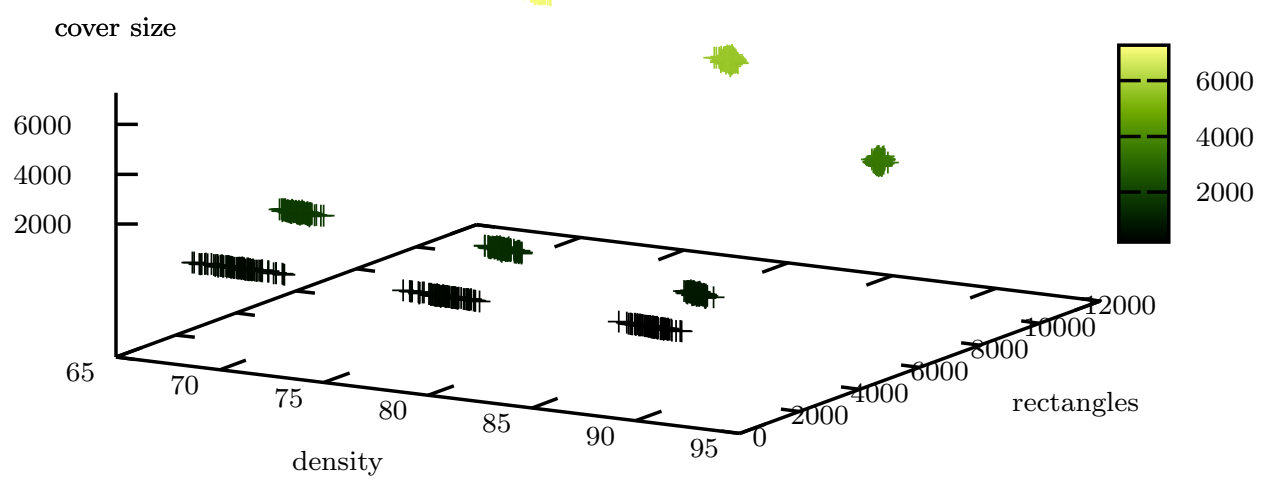

Fig. 7. Every cross in this and the following pictures represents a single random instance. This picture visualizes the very low combinatorial complexity of random flip-coin type polygons: Knowing the number of rectangles in an such a polygon almost allows guessing the size of a greedy cover.

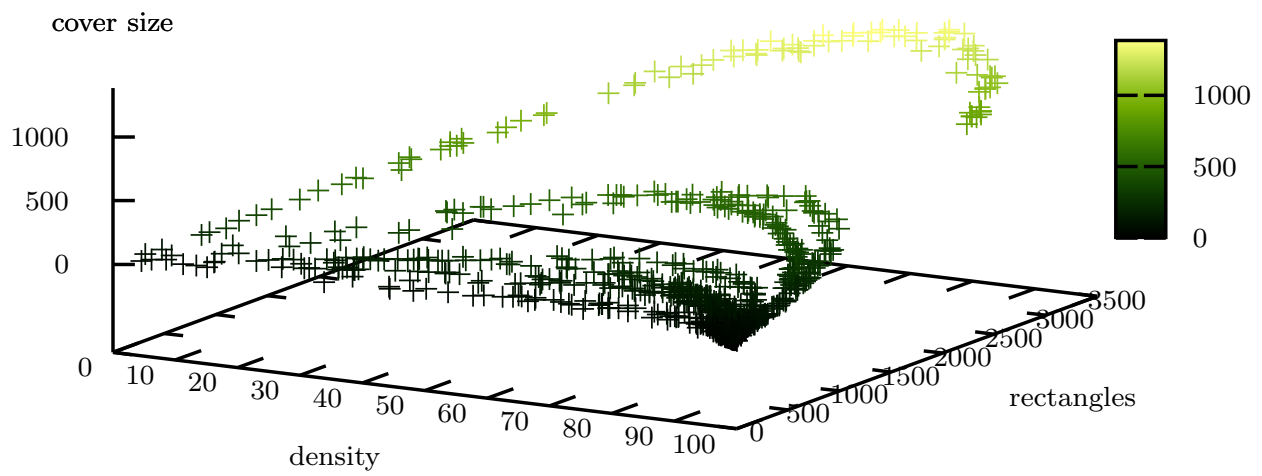

Fig. 8. Combinatorial complexity of random union type polygons. At a medium density around $50 \%$ a cover uses the largest fraction of available rectangles. 


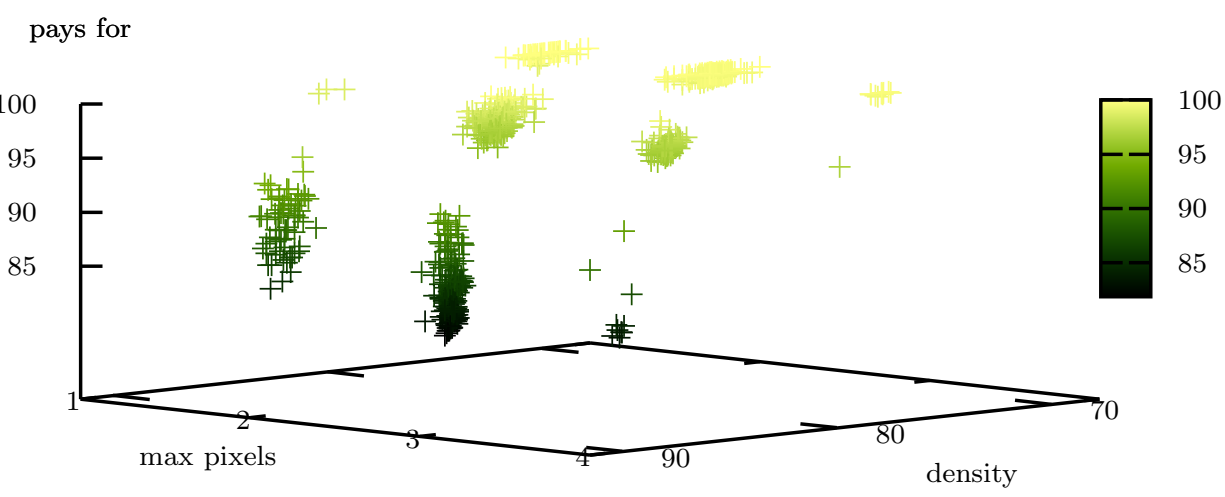

Fig. 9. Quasi-Greedy's computation of pseudo stable sets on flip-coin instances. The axis labels correspond to the headings in Tables II and IV. Denser polygons make it harder for a pseudo stable set to pay for the whole cover. The number of pseudo stable pixels in some rectangle never exceeded 4.

pays for

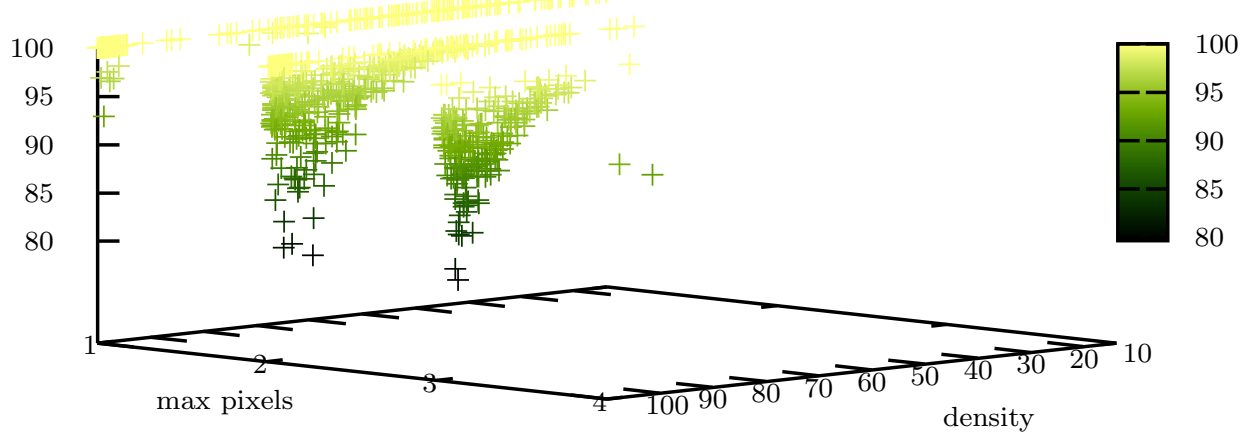

Fig. 10. This is Fig. 9 for union instances. 


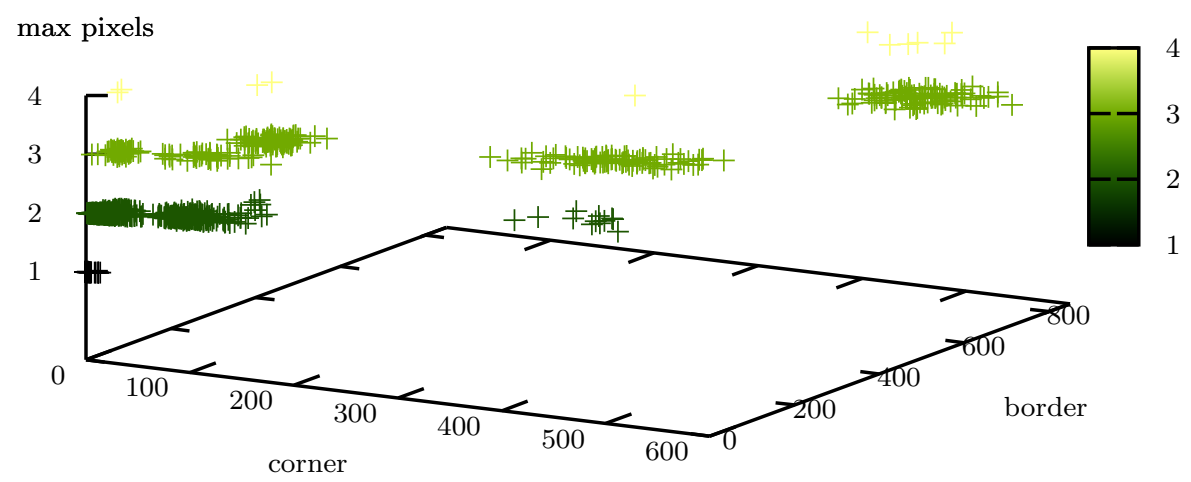

Fig. 11. Quasi-Greedy's computation of pseudo stable sets on flip-coin instances. The need to use border and corner pixels in a pseudo stable set increases chances that more pseudo stable pixels are contained in some rectangle, as was to be expected.

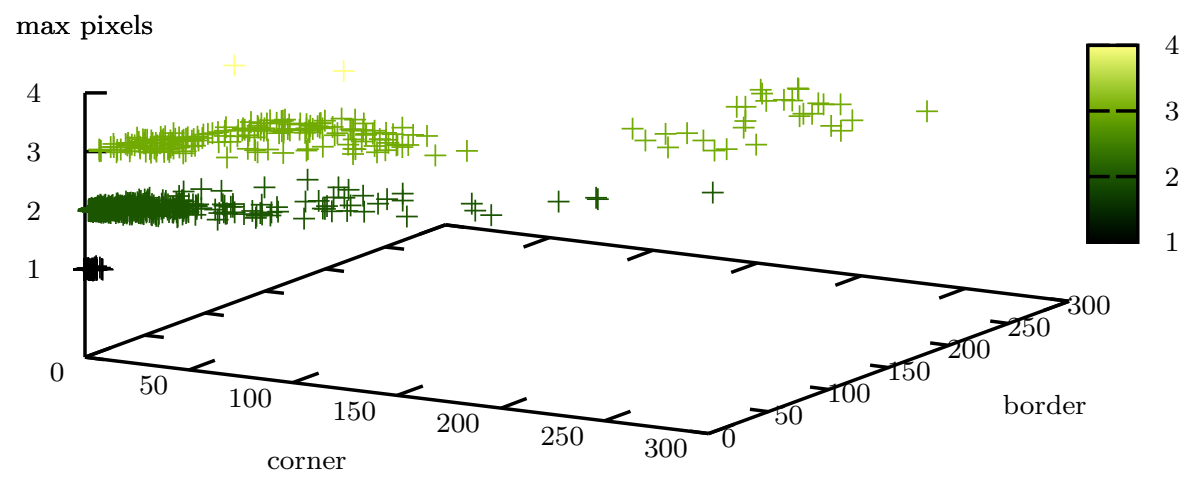

Fig. 12. This is Fig. 11 for union instances. 
pays for

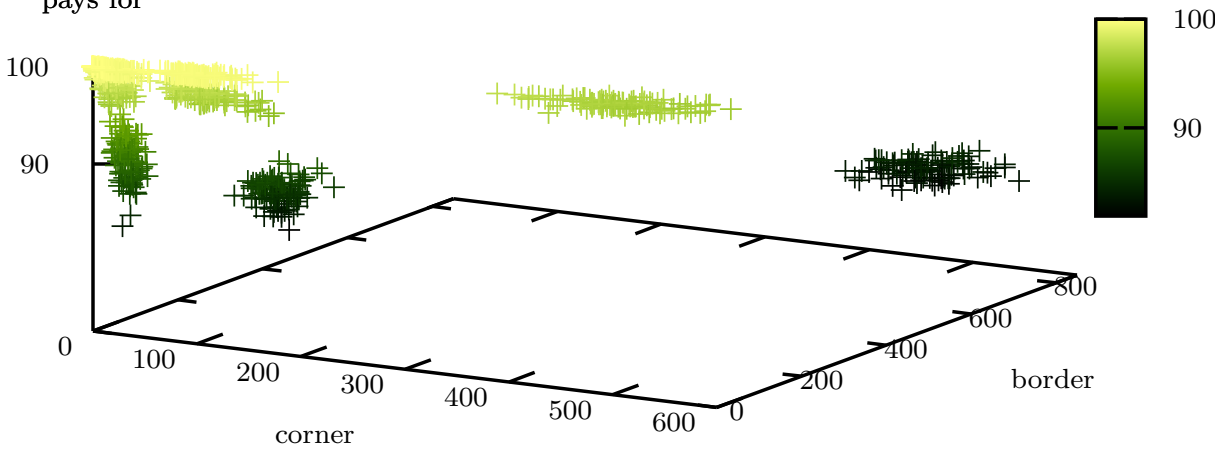

Fig. 13. QuASI-GREeDY's computation of pseudo stable sets on flip-coin instances. This is the analogue to Fig. 11, evaluating the fraction of the cover the pseudo stable set pays for. Here again, the need to use border and corner pixels decreases chances that the pseudo stable set is able to pay for the whole cover or a very large fraction of it.

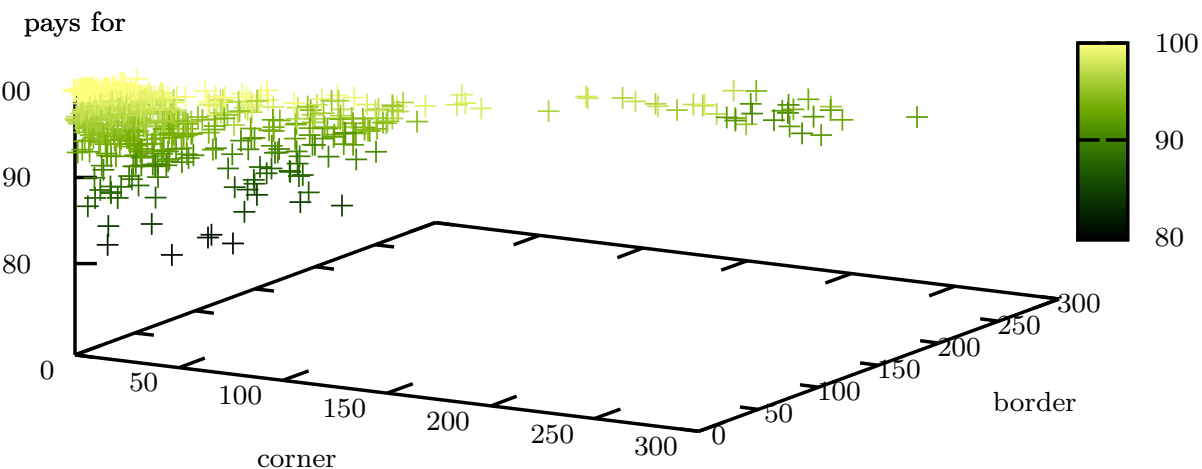

Fig. 14. This is Fig. 13 for union instances. 